\title{
De la neuroplasticidad a las propuestas aplicadas: estimulación temprana y su implementación en Costa Rica

\author{
From Neuroplasticity to Applied Proposals: Early Stimulation and Its \\ Implementation in Costa Rica
}

\author{
Johanna Sibaja-Molina, Tracy Sánchez-Pacheco, \\ Mijail Rojas-Carvajal y Jaime Fornaguera-Trías \\ Universidad de Costa Rica, Costa Rica
}

\begin{abstract}
Resumen
La discusión en torno a la influencia del ambiente sobre la ontogenia ha alimentado la interrogante sobre cuál es el grado en el que, a través de la manipulación del contexto, se modifican las características de los individuos. Actualmente, la evidencia científica desde distintos campos (e.g., Psicología del desarrollo, Neurociencias) describe cómo el ambiente es capaz de modular los procesos del desarrollo y las distintas capacidades del cerebro, así como los mecanismos plásticos que subyacen a dicha modulación. Con base en esta evidencia, distintas aproximaciones metodológicas orientadas a la estimulación temprana (ET) se han propuesto potenciar el desarrollo o remediar problemas que se presentan durante las etapas tempranas del ciclo vital. En este contexto, se brinda una revisión del tema y se ofrece un marco teórico general sobre los antecedentes y sobre los principios que sustentan la estimulación del sistema nervioso (i.e., plasticidad cerebral). Además, a través de una aproximación empírica y un proceso de revisión bibliográfica, se presenta la evidencia disponible de algunos de los métodos de mayor conocimiento/uso en Costa Rica (i.e., DomanDelacato, Snoezelen ${ }^{\circledR}$, Point y Bebé Políglota). Al recapitular los alcances y las limitaciones de la ET, se concluye que es necesario sistematizar las experiencias profesionales de forma tal que permitan abrir un debate académico sobre el tema.
\end{abstract}

Palabras clave: Estimulación temprana, plasticidad, neurodesarrollo, Doman-Delacato, Snoezelen ${ }^{\circledR}$, Método Point y Bebé Políglota

Johanna Sibaja-Molina, Centro de Investigación en Neurociencias, Escuela de Psicología, Escuela de Formación Docente, Universidad de Costa Rica; Tracy Sánchez-Pacheco, Centro de Investigación en Neurociencias, Instituto de Investigaciones Psicológicas, Universidad de Costa Rica; Mijail Rojas-Carvajal, Centro de Investigación en Neurociencias, Universidad de Costa Rica; Jaime Fornaguera-Trías, Centro de Investigación en Neurociencias, Departamento de Bioquímica, Escuela de Medicina, Universidad de Costa Rica.

La correspondencia en relación con este artículo se dirige a Johanna Sibaja-Molina, dirección electrónica: johanna.sibajamolina@ucr.ac.cr 


\begin{abstract}
The discussion about environmental influence on ontogeny has fueled the question about how much context manipulation can regulate individuals' characteristics. Today, scientific evidence from different fields (e.g., developmental psychology, neuroscience) describes not only how environment can modulate brain development and function, but also the plastic mechanisms involved. In consequence, different methodological approximations of early stimulation (ES) have arisen, although they are not necessarily based on solid empirical evidence. In this context, a brief approximation to the ES background is provided, as well as a general framework about brain plasticity. In addition, the theoretical and practical perspective of the ES practitioners is also described by detailing the empirical evidence around its most known/used variants in Costa Rica (i.e., Doman-Delacato, Snoezelen $\AA$, Point and Bebé políglota). Finally, bringing up the scope and limitations of ES, we conclude that the professional experiences need to be systematized, in order to open an academic debate on the subject.
\end{abstract}

Keywords: Early stimulation, plasticity, neurodevelopment, Doman-Delacato, Snoezelen ${ }^{\circledR}$, Point method, Polyglot world $®$

La Psicología del desarrollo estudia los cambios de las personas a lo largo del tiempo a partir de tendencias normativas generalmente relacionadas con la edad (Palacios, Marchesi, \& Coll, 2006). Producto de la investigación científica en esta área, se ha podido caracterizar, y muchas veces explicar, la complejidad subyacente al desarrollo humano: actualmente es de carácter multidireccional y atiende a dimensiones biológicas, contextuales e históricas (Bronfenbrenner \& Morris, 2006; Palacios et al., 2006); es decir, el desarrollo debe entenderse más allá del resultado de un despliegue madurativo, en donde la interacción sujeto-medio modula la adquisición de habilidades para la flexible y la compleja manipulación de información (Bronfenbrenner \& Morris, 2006).

Gracias al progreso en el entendimiento sobre el desarrollo ontogenético, se han generado diversas aproximaciones que buscan potenciar sus distintos procesos, o bien, remediar aquellos que ocurren de forma atípica. Una de estas aproximaciones es la estimulación temprana (ET), cuyo objetivo consiste en promover la salud y bienestar del infante, reforzar competencias emergentes, minimizar los retrasos en el desarrollo, así como promover un adecuado funcionamiento de la familia (Bonnier, 2008; Shonkoff $\&$ Meisels, 2000). En la actualidad, se ofrece una amplia gama de métodos de ET dentro de contextos educativos y de crianza en Costa Rica. Se infiere que el interés por la incorporación de la ET dentro de la educación inicial responde, en parte, a la necesidad de ofrecer intervenciones más tempranas en procesos de desarrollo que se encuentran comprometidos. Desde una perspectiva histórica, también resulta posible entender a la ET como una forma de educación extra-familiar orientada a promover el cuidado y hábitos básicos, así como a realzar el valor de la niñez y cultivar sus destrezas. Dichos intereses se han forjado a partir de contextos socioeconómicos en los que se requiere la incorporación de las mujeres a la vida laboral fuera del hogar (Chavarría-González, 1982).

En las sistematizaciones sobre el tema, la premisa básica que supone sustentar la efectividad de la ET es la "plasticidad" de las personas durante los primeros años de vida. Se asume, entonces, que 
realizar intervenciones a temprana edad generará efectos positivos en el desarrollo. De esta manera, las referencias teóricas o empíricas disponibles suelen enfocarse en el diseño o implementación de programas de ET al realizar un análisis a nivel general. Cabe destacar que en el país no hay datos publicados que permitan visualizar quiénes son el público meta de estos servicios, los motivos de consulta, quiénes están llevando a cabo su implementación, las ventajas que suponen los programas, los instrumentos para valorar los cambios generados por la ET, y sobre todo, cuáles son las bases empíricas que apoyan cada uno de los métodos que se ofrecen.

Este artículo busca establecer un marco de referencia que permita comprender el estado actual de la implementación de la ET en el contexto costarricense. Lo anterior al considerar el impacto que las experiencias tempranas tienen en el desarrollo humano, así como la perspectiva educativa, histórica y académica de la Psicología del desarrollo en nuestro país (Tapia, Carmiol, \& Rosabal-Coto, 2012). Para ello, se realizó una búsqueda de centros infantiles en los que se ofrecieran servicios de ET dentro del área metropolitana en Costa Rica. Una vez identificados, se contactó a los profesionales de esos centros y se les aplicó una encuesta para determinar cuáles son los métodos de ET que más se conocen y que más se utilizan en el país, así como para detectar la población a la que se dirigen los servicios, los motivos por los que se accede, las ventajas que suponen y los instrumentos que se utilizan para valorar los cambios. A partir de la información obtenida, se realizó una revisión bibliográfica sobre la evidencia empírica de los métodos más mencionados en dicha encuesta.

El artículo, además, ofrece un breve contexto del origen de la ET, así como los procesos neurobiológicos en los que supone sustentarse con el objetivo didáctico de brindar insumos para la formación de criterio de los y las lectoras. Así, este trabajo pretende contribuir a la sistematización sobre el tema con el fin de propiciar un debate académico, tanto a nivel conceptual como metodológico.

\section{Contextualización breve de los orígenes de la Estimulación Temprana}

A raíz de los conflictos bélicos de finales del siglo XIX e inicios del XX, distintas organizaciones educativas, públicas y privadas desarrollaron procedimientos didácticos y de cuidado físico para compensar los efectos nocivos de la negligencia parental y las hambrunas (Kamerman, 2006). Esta preocupación por el impacto negativo de las experiencias tempranas adversas surge a partir de los estudios en orfanatos, en donde la carencia de interacciones cálidas, íntimas y continuas de los infantes con sus cuidadores mostró ser un indicador negativo de salud mental (Bowlby, 1952). Desde entonces, diversas iniciativas se orientaron a generar estrategias de intervención particularmente dirigidas a la recuperación de trastornos o deficiencias, tanto sensoriomotoras como cognitivo-emocionales (Kamerman, 2006). Muchas de las cuales se apoyaron en campos como la educación, servicios de salud maternoinfantiles, la educación especial e investigación sobre desarrollo infantil (Shonkoff \& Meisels, 2000).

Los procesos de investigación empírica sobre el tema generaron un sustento teórico para conocer no sólo las secuelas de los ambientes adversos sino también las condiciones en las que estas podrían revertirse (Shonkoff \& Meisels, 2000). Esta evidencia se acompañó de resultados en modelos animales, que indagaban sobre la plasticidad cerebral y el efecto compensador de la "estimulación" a distintos niveles (Rosenzweig, Bennett, \& Diamond, 1972; Sale, Berardi, \& Maffei, 2014). 
Desde la perspectiva psicológica, los desarrollos teóricos y empíricos de Piaget y Bowlby sirvieron como base para determinar el carácter progresivo y escalonado de distintos procesos del desarrollo psicomotor, cognitivo y socioemocional. A su vez, el trabajo de Vigotsky fue fundamental en cuanto al señalamiento de la relevancia que tienen espacios y tiempos específicos que permiten dicho desarrollo (Palacios et al., 2006). Todos estos aportes se conjugaron para concebir el desarrollo temprano como un período altamente prolífico y maleable.

En este sentido, los modelos de intervención y estimulación partieron de la premisa de que el sistema nervioso es capaz de modificarse y adaptarse como producto de la experiencia y que esta capacidad es mayor durante la niñez (Sale et al., 2014; Baker-Henningham, \& López-Boo, 2014). Lo anterior ha llevado a que la ET trascienda la esfera del intervencionismo en condiciones atípicas de desarrollo y se incorpore como una práctica orientada a la niñez en general. A pesar de que la idea de la flexibilidad o plasticidad del cerebro precede la aparición de estos abordajes (Ghalambor, McKay, Carroll, \& Reznick, 2007; James, 1890), sus alcances son actualmente un tema en desarrollo. Se describe, a continuación, un marco de referencia conceptual sobre la plasticidad cerebral o neuroplasticidad, así como sobre los mecanismos neurobiológicos subyacentes.

\section{Plasticidad y epigenética en el Sistema Nervioso Central: sustrato neurobiológico de los efectos del ambiente sobre los individuos}

\section{Conceptos de plasticidad}

En la vida cotidiana, se enfrentan constantes desafíos que requieren respuestas flexibles. En este contexto, la capacidad del encéfalo para almacenar y procesar información permite generar nuevos patrones de respuesta que facilitan una adaptación rápida y duradera al ambiente (Ghalambor et al., 2007; James, 1890). Esta capacidad para experimentar cambios funcionales, estructurales y morfológicos en función de la experiencia se conoce como plasticidad cerebral (Hebb, 1949; Kandel, Schwartz, Jessel, Siegelbaim, \& Hudspeth, 2013; Kolb, Gribb, \& Robinson, 2003; Kolb \& Wishaw, 1998). A este respecto, se describirán los distintos niveles en los que ocurre, así como su participación en los procesos de ET.

\section{Cambios funcionales}

La plasticidad, en términos funcionales, se refiere a la capacidad de los organismos para aprender y modular sus respuestas en función de la adquisición y transformación de nueva información (Abraham, Vincis, Lagier, Rodríguez, \& Carleton, 2014; Kolb et al., 2003). Por ejemplo, un cambio funcional refiere a la posibilidad de asociar un estímulo con una respuesta que, en principio, no se encontraban asociados (Kandel et al., 2013; Abraham et al., 2014). Como es de suponerse, existen mecanismos neuronales subyacentes que proporcionan su sustrato y, a la vez, limitan su rango de cambio.

\section{Reconfiguración de la membrana celular y funciones asociadas}

Los cambios funcionales se encuentran asociados con modificaciones en las propiedades de las membranas y en otras funciones relacionadas con la transmisión de la señales entre las neuronas (Hebb, 1949; Kandel et al., 2013). Cuando el disparo de una neurona "A" cocurre con el disparo de una neurona "B", tal que A facilita el disparo de B, se establece una relación entre la probabilidad de disparo de esas neuronas (Hebb, 1949); es decir, ambas "aprenden" a disparar juntas. Este proceso surge por el cambio 
en la distribución de las proteínas encargadas de recibir la señal entre neuronas, a saber, los receptores (específicamente, los receptores AMPA; Lüscher \& Malenka, 2012). La activación conjunta de A y B genera un incremento en el número de receptores en B (Brady, Siegel, Albers, \& Price, 2012; Lüscher \& Malenka, 2012). Gracias a esto, se incrementa la sensibilidad de la neurona possináptica (B) a las señales de la neurona presináptica (A), generando una asociación entre ellas; proceso al que se le conoce como potenciación a largo plazo (PLP; Brady et al., 2012; Lüscher \& Malenka, 2012).

\section{Cambios en la morfología celular}

Las células nerviosas experimentan diversos tipos de cambios que facilitan el aprendizaje y la memoria (en múltiples ámbitos) justo después de su adquisición, también durante etapas posteriores. Horas después de la PLP, se produce un incremento en el número de espinas dendríticas, que son pequeñas prolongaciones de las dendritas, que constituyen uno de los sitios de recepción de información de las neuronas (Engert \& Bonhoeffer, 1999). De hecho, el área de neurotransmisión de la dendrita se expande y se divide durante las fases tardías de la PLP, hasta que posteriormente una sola sinapsis (conexión espina dendrítica-axón) se transforma en dos o más (Geinisman, 2000). Coincidentemente, en modelos animales se ha descrito que el entrenamiento en ambientes espacialmente complejos incrementa el número de espinas dendríticas en el hipocampo (región del encéfalo vinculada con el procesamiento de información espacial y la memoria), lo cual se asocia con un mejor desempeño en tareas de memoria espacial (Moser, Trommald, \& Andersen, 1994). En este caso, la plasticidad se evidencia en la capacidad de las células nerviosas para modificar sus conexiones existentes, o bien, establecer nuevas conexiones.

\section{Interacción herencia/medio sobre la plasticidad cerebral}

Los cambios neurofisiológicos descritos son el resultado de la interacción de los sujetos con el medio y las respuestas cognitivas, emocionales y conductuales son su "producto funcional". En este sentido, el genotipo (información genética que contiene un organismo) constituye solo una variable que explica al fenotipo (características observables) final de los individuos y la interacción con el ambiente constituye una de las más importantes fuentes de modulación (Rutter, 2007). Pensemos en un juego de bloques, en donde el conjunto de sus piezas constituye el genotipo. Estos bloques representan la materia prima que determinará en cierta medida las construcciones por realizar; sin embargo, las posibles combinaciones que se realicen con los bloques serán en gran medida influenciadas por quien construye (ambiente e interacción ambiente-genes). Tanto el acervo genético (características de los bloques) restringe el rango de posibles variaciones, como el ambiente (versatilidad de quién construye) influencia cuáles de esas variaciones se expresan, es decir, el fenotipo (Rutter, 2007). Esta modificación del fenotipo producto de la regulación de la expresión del genotipo a menudo ocurre mediante mecanismos epigenéticos.

\section{Mecanismos epigenéticos de regulación de la plasticidad cerebral}

La epigenética ha sido fundamental para entender cómo la experiencia se traduce en cambios celulares. Su estudio comprende la modificación en la expresión génica no asociada con cambios en la secuencia de ADN (Mifsud et al., 2011; Sweatt, 2009). Los genes pueden estar o no disponibles para ser transcritos, en donde la experiencia (p.e. aprendizaje), estímulos ambientales y el consumo de sustancias, modifican su disponibilidad y se logra, finalmente, modular la expresión génica (Sweatt, 2009). Una de las formas en que la experiencia genera estos cambios es mediante la PLP, porque activa distintas vías de 
señalización que aumentan o disminuyen la accesibilidad de ciertos genes, lo cual determinaría su estado de activación/silenciamiento (Giese \& Mizuno, 2013). Estos cambios en la expresión génica se traducen en la función o estructura de la neurona, o bien, facilitan la supervivencia de nuevas neuronas (Giese \& Mizuno, 2013; Sweatt, 2009). Este es solo uno de los mecanismos de la plasticidad asociado con la experiencia, el cual brinda una idea sobre la existencia de procesos moleculares y bioquímicos especializados en transformar las señales del ambiente en cambios neuronales funcionales.

\section{Neuroplasticidad y estimulación temprana}

La ET parte del concepto de plasticidad para introducir una variedad de estímulos dirigidos a generar cambios observables en los sujetos. Si bien es cierto que su impacto positivo sobre la ontogenia ha sido ampliamente difundido (Kamerman, 2006), los estudios se han circunscrito principalmente a sujetos con desarrollo no normativo, tal como en casos de personas con síndrome de Down, niños prematuros, parálisis cerebral y sobre características específicas tales como el desarrollo motor (Sale et al., 2014). La evidencia que cuenta con mayor solidez, al respecto, se refiere al estudio en modelos animales (ratas) de enriquecimiento ambiental (EA), en donde se ha observado una potenciación de sus capacidades para el aprendizaje, la memoria y el control emocional por sobre sujetos criados en condiciones estándar de laboratorio (ver Deng, Aimone, \& Gage, 2010; Mora-Gallegos et al., 2015). Dentro de esta misma línea, también se ha identificado que existen condiciones de estimulación que no generan efectos favorables. Por ejemplo, ratas enriquecidas que solo fueron estimuladas mediante actividad física atendieron en menor proporción a llamados de contacto social, en comparación con las que fueron expuestas a estimulación física y social simultáneamente (Brenes et al., 2016). Además, ratones que fueron estimulados solo mediante actividad física muestran una disminución en los niveles de exploración y contacto con nuevos ambientes y estímulos (Pietropaolo et al., 2004). Si bien, los animales enriquecidos mediante actividad física mostraron mejor desempeño en otros dominios al compararlos con grupos no enriquecidos, esta evidencia sugiere que existe un efecto diferencial según el tipo de estimulación.

Dado que los distintos métodos de ET pretenden utilizar como justificación los principios de neuroplasticidad (Doman \& Doman, 2006; Editorial Baby Star, 2009; Hotz et al., 2006), se considera relevante indagar sobre la evidencia empírica con la que cuentan estas variantes. Con ese objetivo, se realizó una consulta a profesionales que utilizaban métodos de ET, en la que se incluyeron diferentes preguntas que se detallarán más adelante.

\section{Metodología del estudio}

Mediante el motor de búsqueda Google y utilizando las palabras clave "estimulación temprana" $A N D$ "Costa Rica" se realizó el rastreo de profesionales y de centros de atención infantil que ofrecieran servicios de ET dentro del área metropolitana en Costa Rica. Se identificaron 46 contactos, 30 de los cuales accedieron a participar. Posteriormente, con el fin de ampliar la muestra, se solicitó la colaboración abierta a través de la cuenta del Centro de Investigación en Neurociencias de la Universidad de Costa Rica en Facebook. El resultado fue la colaboración de 14 personas más. Una vez establecido el contacto con todas las personas interesadas, se les entregó la encuesta de forma física o digital, según su preferencia. 


\section{Participantes}

Un total de 44 participantes contestaron por completo el documento. En su totalidad eran mujeres del gran área metropolitana, cuyas edades oscilaron entre los 23 y los 58 años $(M=32.5, D E=7.18)$.

\section{Instrumentos}

El instrumento contó con preguntas abiertas. Las respuestas brindadas se clasificaron en categorías según su contenido. Se reporta el porcentaje de respuestas agrupadas por categoría, como unidad de medida. En este sentido, el porcentaje no corresponde al número de profesionales entrevistadas, sino al número de respuestas emitidas y agrupadas (consultar detalles de la encuesta en http://hdl.handle.net/10669/15644).

A partir de los resultados de las encuestas, se seleccionaron algunos de los métodos más frecuentes para investigar la evidencia empírica que les respalda. Para ello, se consultaron bases de datos científicas (e.g., EBSCO, ERIC, JSTOR, Redalyc, Science Direct, Springer), buscadores especializados (e.g., Google académico, PubMed), y portales de difusión científica (e.g., Dialnet). Asimismo, se utilizaron las bases de datos ofrecidas por la Universidad de Costa Rica (e.g., SIBDI). Los criterios de inclusión de los artículos seleccionados fueron los siguientes: (a) ser publicados en revistas que cuenten con revisión por pares, (b) incluir como palabra clave el nombre del método y (c) contar con población infantil como participante del estudio. En caso de no obtener resultados, se ampliaron los criterios de inclusión y los motores de búsqueda, lo cual se especifica en los apartados respectivos.

\section{Resultados}

De las personas que respondieron a la encuesta, el 5\% reportó ser estudiante de bachillerato universitario, el 14\% reportó contar con bachillerato universitario, el $43 \%$ con grado licenciatura y el 36\% con maestría. El rango de años de experiencia realizando ET es de cero hasta 22 años $(M=5, D E=4.90)$. El 82\% reportó haber recibido capacitación o educación formal en el tema, del cual un $44 \%$ recibió dicha formación en la Universidad Santa Paula.

Del proceso exploratorio, sobre la situación de la ET, se detallan los siguientes porcentajes:

\section{¿A quién se dirigen los servicios de estimulación temprana?}

Al consultar a las especialistas sobre cuál es la población a la que se dirige la ET, en el $61 \%$ de las respuestas se indicó que son servicios dirigidos a cualquier niño o niña menor de siete años. Un 20 $\%$ de las respuestas describió que la ET se dirige exclusivamente a niños y niñas con alguna necesidad educativa especial o retraso en el desarrollo. Mientras que en un $6 \%$ se señaló que estos servicios suelen estar dirigidos a infantes provenientes de una clase social media-alta.

\section{¿Cuáles son los motivos de consulta en estimulación temprana?}

En un 50\% de las respuestas se asegura que el motivo de consulta más común es el interés de las familias por potenciar el desarrollo de niños y niñas. A lo interno de esta categoría, un $32 \%$ de las respuestas asegura que el interés de esa potenciación se dirige, principalmente, al aumento de la capacidad intelectual y un $12 \%$ al interés de las familias por lograr que el infante despliegue habilidades por encima del promedio. El segundo motivo más mencionado abarca el $34 \%$ de las respuestas y refiere al temor de 
las familias de que sus hijos presenten algún retraso en el desarrollo. A lo interno de esta categoría, un 59 $\%$ asegura que sus consultantes fueron referidos por un especialista.

\section{¿Cuáles ventajas supone la estimulación temprana}

Un $12 \%$ de las respuestas describe una mejora en los procesos de socialización, un 11\% apunta a un favorecimiento de las habilidades de razonamiento y resolución de problemas, un $10 \%$ dice haber observado un aumento en el autoconocimiento y la seguridad en sí mismos, un $9 \%$ hizo referencia a un beneficio en la autorregulación emocional, así como un aumento en las competencias de comunicación (9\%) y una mejora en las habilidades sociales $(9 \%)$.

\section{Conocimiento de instrumentos utilizados para valorar la efectividad de la estimulación temprana}

Solo el $43 \%$ de las participantes asegura conocer o utilizar algún tipo de instrumento para evaluar la efectividad de las intervenciones de ET que realizan. Los instrumentos mencionados con mayor frecuencia son escalas y pruebas de desarrollo, dentro de las que se encuentra el test de Denver (Frankenburg \& Dodds, 1967), la tabla de desarrollo de Haizea-Llevant (Fernández, Fuente, \& Rueda, 1991), el test de desarrollo psicomotor (TEPSI, Haeussler \& Marchant, 1985), el Manual Operativo para la Evaluación y Estimulación del Crecimiento y Desarrollo del Niño (Hernández \& Rodríguez, 1987), la escala de evaluación del desarrollo Escala de Desarrollo Integral (EDIN; Atkin, Superville, Sawyer, \& Cantón, 1987), y la guía Portage de Educación Preescolar (Bluma, Shearer, Forman, \& Hillard, 1978). Además, se mencionaron instrumentos para evaluar áreas del desarrollo específicas tales como el Test Wepman (Wepman, 1958) para evaluar lenguaje y la Escala Motriz del Infante de Alberta (Piper \& Darrah, 1994) para evaluación del área motora. Se reportó el uso de cuestionarios e instrumentos de elaboración propia.

Reporte sobre métodos de estimulación temprana: Descripción y respaldo empírico de sus resultados

Dentro de los métodos de ET más reportados por las especialistas están los siguientes: DomanDelacato (16\%), Snoezelen (6\%), Point (6\%) y Bebé Políglota (4\%). El porcentaje restante se distribuyó en respuestas varias, muchas de las cuales no categorizan como métodos de ET.

\section{Método Doman-Delacato}

Este método fue elaborado entre los años 1956 y 1957 con el fin de atender a infantes con lesiones cerebrales (Doman, Spitz, Zucman, Delacato \& Doman, 1960), y partió del desarrollo de su propia teoría de organización neurológica. Esta fue influenciada por la propuesta de recapitulación filogenética de Ernst Haeckel, la cual sostiene que la ontogenia sigue el mismo patrón de desarrollo que la secuencia filogenética de la especie a lo largo de su evolución (MacKay, Gollogly, \& McDonald, 1986; Vergara-Díaz, MartínezGalán, Martínez-Sahuquillo, \& Echevarría-Ruiz, 2011; Wachelke, Natividade, Faggiani, \& De Andrade, 2004). Así, los casos de retraso mental, problemas de aprendizaje y trastornos de la conducta se explican como el resultado de un daño en alguna de las etapas de esa evolución, lo que supone una organización neurológica inadecuada (Ziring et al., 1999).

La teoría sostiene que el desarrollo de los procesos cognitivos superiores depende de la ejecución escalonada de movimientos corporales primitivos a lo largo de la infancia temprana (Hornby, Atkinson, \& Howard, 2013); es decir, de no realizarse correctamente se corre el riesgo afectar el desempeño 
cognitivo (Glass \& Robbins, 1967; Keshner, 1967). Para abordar estos casos, Doman et al., (1960) establecen programas de movimientos conocidos como "patterning", cuyo objetivo es la reorganización funcional del sistema nervioso a través de la ejecución repetida de patrones motores.

En sus anotaciones anecdóticas, Doman describe cómo los niños con lesiones cerebrales progresaban gracias a los programas de intervención y mostraron comportamientos similares o mejores a los de niños promedio. Producto de su experiencia, se cuestiona si los niños con desarrollo normativo estaban siendo subestimados en su potencial de aprendizaje. Por ello, decide sistematizar su práctica clínica y educativa al trasladarla a una población con desarrollo normativo con el fin de potenciar sus capacidades por encima del promedio (Doman \& Doman, 2006).

Esta sistematización de experiencias da paso a una serie de programas secuenciales en donde se fomenta la adquisición temprana de lectura, matemática, conocimientos enciclopédicos, idiomas y música. Lo anterior con base en la idea de "bits de inteligencia", que alude a un aprendizaje mediante la exposición breve y repetida a pequeñas unidades de información (Chaparro, 2010).

La propuesta de intervención sugiere que debe realizarse preferiblemente desde los cero años. Se inicia como estimulación visual; de no ser así, el método ofrece variaciones (Doman \& Doman, 2006). Los autores son enfáticos en la necesidad de comenzar la intervención lo antes posible. Se parte de la metáfora de que los niños son como una computadora vacía a la que hay que llenar de información (Doman \& Doman, 2006).

El método fue ampliamente criticado desde su propuesta inicial. No solamente por la carencia de evidencia científica (American Academy of Pediatrics, 1968), sino también por los efectos adversos que mostraba su implementación (Bratt, 1989; Glass, 1968; Vergara-Díaz et al., 2011). Una de las críticas más fuertes fue el haberse valido de una generalización para ignorar diferencias etiológicas en los diagnósticos, porque se aseguraba la cura a condiciones diagnósticas como sordera y ceguera (The Institutes for the Achievement of Human Potential, 2016), que en su amplio espectro son tratables pero no reversibles (Wachelke et al., 2004). Es precisamente, por esto, que tanto profesionales (Cruickshank, 1968) como instituciones (American Academy of Pediatrics, 1968; American Academy of Pediatrics, 1999) se pronunciaron en contra del método. De hecho, revisiones bibliográficas detalladas han concluido que la mayoría los estudios empíricos sobre el método presentan errores metodológicos claros que comprometen la capacidad de generalización de los resultados (Vergara-Díaz et al., 2011; Wachelke et al., 2004; Glass \& Robbins, 1967), tales como la inexistencia de grupos control (Doman et al., 1960), la no asignación aleatoria a los grupos de intervención (Kershner, 1967), la no homogeneidad en las condiciones de aplicación del tratamiento entre grupos (Stone \& Pielstick, 1969) y la deficiencia de sus análisis estadísticos (Delacato, 1959; Doman et al., 1960).

\section{Método Snoezelen ${ }^{\circledR}$}

En 1975, se desarrolló la idea de los espacios Snoezelen® (Hulsegge \& Verheul, 1988), cuyo objetivo fue brindar a adultos institucionalizados, por discapacidad mental, un espacio recreativo que involucrará dinámicas multi-sensoriales (Hulsegge \& Verheul, 1988). En estos ambientes se fomenta la exploración libre del usuario sobre distintos estímulos visuales, auditivos, táctiles, olfatorios y propioceptivos, acompañado de forma no directiva por un facilitador (Fowler, 2008; Haegele \& Porretta, 2014; Hulsegge \& Verheul, 1988). Los espacios Snoezelen ${ }^{\circledR}$ se caracterizan por la combinación de luces, 
sonidos, aromas y texturas, cuyo objetivo es generar una experiencia agradable y de relajación en los participantes (Haggar \& Hutchinson, 1991).

Su utilización se ha extendido a diferentes países, entre ellos Costa Rica (Rojas, 2014), y cada vez son más las escuelas, centros recreativos, hospitales y clínicas que lo implementan (Bozic, 1997; Haegele \& Porretta, 2014; Shapiro, Melmed, Sgan-Cohen, Eli, \& Parush, 2007).

Se le han atribuido propiedades terapéuticas, bajo el razonamiento de que la frecuencia y variedad de estímulos propicia el crecimiento dendrítico y mejora las conexiones sinápticas, sobre todo en casos en que existe daño en el sistema nervioso (Hotz et al., 2006).

Como enfoque terapéutico, ha sido aplicado en escuelas de educación especial para apoyar a infantes con discapacidades severas (Stephenson \& Carter, 2011), al igual que en el abordaje terapéutico de casos con síndrome de Rett (Lotan \& Shapiro, 2005), retardo mental (Shapiro, Parush, Green, \& Roth,1997), daño cerebral (Hotz et al., 2006), discapacidades intelectuales (Nasser, Cahana, Kandel, Kessel \& Merrick, 2004) y otras discapacidades en el desarrollo (Lancioni, Cuvo \& O’Reilly, 2002; Fowler, 2008).

Algunos estudios han procurado sistematizar las experiencias y evidenciar la efectividad del método al atribuirle la capacidad de disminuir la agitación y la frecuencia cardíaca, lo cual se asocia con estados de relajación (Hotz et al., 2006; Lotan \& Shapiro, 2005; Nasser et al., 2004). También, se reporta una disminución de la frecuencia y la duración de conductas desadaptativas durante las sesiones en la sala Snoezelen $\mathbb{}$ en comparación con las sesiones en una sala de juego (Shapiro et al., 1997) y, además, se muestra una mejoría en los procesos de concentración (Botts, Hershfeldt, \& Christensen-Sandfort, 2008).

Si bien el método cuenta con numerosas investigaciones, aún la evidencia no permite corroborar su efectividad debido a deficiencias metodológicas, como la excesiva dependencia de la evaluación cualitati$\mathrm{va}$, la ausencia de condiciones control, las imprecisiones operacionales de las conductas meta y la ausencia de fiabilidad de las mediciones reportadas en los estudios (Hogg, Cavet, Lambe, \& Smeddle, 2001). También, debido a la heterogeneidad de los diseños de investigación y al reducido número de participantes en los estudios (Lotan \& Gold, 2009). Por lo tanto, desde la evidencia científica, resulta difícil aseverar las ventajas o la eficacia que pueda tener en poblaciones con desarrollo normativo, o bien conocer en detalle los alcances que puede tener su implementación en ambientes escolares convencionales (Stephenson \& Carter, 2011).

\section{Método Point de ET}

El método Point se ha descrito como una estrategia educativa, y como un método de estimulación social, sensoriomotora, cognitiva y artística, dirigido a infantes entre los cero y seis años. Este método tiene como objetivo propiciar el desarrollo de inteligencias múltiples y los procesos de aprendizaje temprano con el fin de garantizar éxito escolar. Además, plantea el aprendizaje natural de una segunda lengua (inglés) y afirma que el bilingüismo aumenta la fluidez y la flexibilidad del pensamiento al utilizar la educación psicomotriz y la estimulación musical para favorecer el desarrollo de la personalidad y mejorar la inteligencia (Terré, 2010). 
A pesar de que se extendió el rastreo de evidencia a motores de búsqueda generales utilizando las palabras clave "método point", "estimulación temprana" AND "método point", "Orlando Terré" (creador del método), no se encontró ninguna referencia sobre investigaciones científicas que respaldaran el método o sus resultados. Además, la literatura reportada y publicada por Terré en su sitio web sobre este método, no fue accesible por medios electrónicos. Tampoco se encontró referencia de investigaciones empíricas que respaldaran las afirmaciones realizadas sobre el método. A pesar de que asegura sustentarse en "concepciones científicas", estas no son descritas ni referidas por su creador en su sitio web oficial (http:// www.orlandoterre.com/metodos.html).

\section{Método Bebé Políglota}

El método Bebé Políglota (BP) se refiere a un programa de estimulación dirigido a niños y niñas menores de siete años. Pretende facilitar el aprendizaje de cinco o más idiomas utilizando estímulos audiovisuales, que incluyen el sonido de locutores nativos hablando cada uno de los idiomas mientras se realizan actividades lúdicas (Ortiz-Alonso, Hall \& García, 2013).

Quienes ofrecen el método, atribuyen la autoría de este programa a Magdalena Granés Morales y afirman que se fundamenta en el método Doman y el método Suzuki para elaborar sus materiales (Leo Global Kids, 2010; Método bebé políglota Costa Rica, 2016b). Aseguran que exponer a infantes a dos sesiones diarias de estimulación de menos de una hora, puede aumentar su capacidad de concentración y memoria (Método Bebé Políglota Costa Rica, 2016a), maximizar su potencial de aprendizaje (Editorial Baby Star, 2009) y aumentar su velocidad de procesamiento cognitivo (Leo Global Kids, 2010).

Dadas las especificaciones de búsqueda de esta revisión, solamente fue posible ubicar un estudio. En este, Ortiz-Alonso et al., (2013) comparan dos grupos de niños y niñas de cuatro años $(M=53.6$ meses, $D E=3.01$ meses) de dos centros educativos bilingües español-inglés. Previo a la intervención, fueron evaluados en una prueba de reconocimiento de vocabulario en cuatro de los idiomas del método (mandarín, portugués, inglés y español), así como la versión española de la escala de inteligencia Weschler (WPPSI-RIII, por sus siglas en inglés). Posteriormente, los infantes de uno de los centros educativos fueron expuestos a dos sesiones diarias del programa BP a lo largo de un año, mientras que el otro grupo fue expuesto a audios de los cuatro idiomas dos meses antes de las siguientes mediciones. Además, se realizó un registro electroencefalográfico mientras los niños completaban las pruebas de reconocimiento de vocabulario.

Se encontró que los niños que estuvieron expuestos al método mejoraron significativamente su capacidad para reconocer palabras, sus puntuaciones en la escala WPPSI-RIII y redujeron las latencias de sus potenciales eléctricos neuronales asociados con el reconocimiento de palabras (Ortiz-Alonso et al., 2013). Sin embargo, el estudio reporta que no hubo asignación aleatoria de los grupos, que desde el inicio el grupo experimental mostró puntuaciones más altas en todas las medidas y contó con más tiempo de exposición al material didáctico. Estas son limitaciones metodológicas que reducen el alcance de las interpretaciones de los resultados, y generan interrogantes sobre si la mejora en sus resultados se debe a diferencias iniciales entre los grupos, a la diferencia entre los tiempos de exposición a los materiales o bien al método en sí mismo. Además, es fundamental entender que la información de un solo estudio no es suficiente para corroborar la eficacia de una intervención desde una perspectiva científica (Crook, Davison, \& Plesser, 2013). 


\section{Discusión}

Desde los estudios clásicos de la Psicología del desarrollo, hasta la evidencia reciente de las Neurociencias, la capacidad de los individuos para cambiar y adaptarse a lo largo del ciclo vital es contundente. Sin embargo, los mecanismos plásticos del sistema nervioso ofrecen restricciones, las cuales deben ser consideradas al aseverar los posibles alcances de la estimulación en contextos de desarrollo normativo. Aunque en la actualidad la ET se utiliza como potenciador intelectual (Editorial Baby Star, 2009; Terré, 2010; Glass \& Robbins, 1967), esta surgió como una herramienta paliativa; es decir, se orientó a recuperar capacidades disminuidas. Por ello, sus efectos sobre el restablecimiento de estas capacidades deben ser interpretados con cautela a la luz de su aplicación en sujetos que no presentan compromisos en el desarrollo.

En el contexto costarricense, se reportó que los servicios de ET se orientan, principalmente, a infantes menores de siete años con desarrollo normativo. La población a la que originalmente se dirigió (e.g., condiciones no normativas en el desarrollo) ocupa en la actualidad un segundo lugar dentro de los motivos de atención. Desde la perspectiva de las especialistas, las principales razones que motivan a las familias a recurrir a la ET son su interés por potenciar capacidades y habilidades en sus hijos e hijas, con la expectativa de observar en ellos un desempeño sobresaliente. En un contexto en el que la divulgación sobre la ET da la impresión de sustentarse científicamente y es insistente en el argumento de lo indispensable de su implementación desde edades tempranas. Entonces, es necesario abrirse al cuestionamiento, sobre todo porque se ha encontrado que el afirmar un sustento científico a una idea incrementa la credibilidad en esta (Weisberg, Keil, Goodstein, Rawson, \& Gray, 2008).

Si bien no es posible negar que la ET ofrece una amplia gama de experiencias sensoriales, físicas y sociales, aseverar que los cambios observados en los infantes son el producto de la implementación de métodos específicos es cuestionable. Para ello, resulta necesario contar con investigación científica de alta rigurosidad metodológica, que permita dilucidar sus efectos, máxime en una etapa del desarrollo donde las variables individuales, socioculturales y el mismo proceso de desarrollo podrían contribuir, en todo o en parte, a explicar dichos cambios. Además, el cambio podría no ser distinto al de infantes cuyas experiencias no se vinculan con métodos de ET.

La generalización y la extrapolación de la evidencia científica desde contextos experimentales controlados hacia ambientes tan distintos como una guardería o un jardín de niños, puede ser problemática. Sin embargo, ha sido evidente que esto ocurre en lo que se refiere a los métodos descritos en el presente trabajo. El afirmar que la exposición a métodos particulares de estimulación durante los primeros años de vida producirá un mejoramiento cognitivo, emocional y social, sin contar con evidencia empírica que lo sustente, además de problemático, podría ser negativo. Esto porque lo que se ofrece del método no concuerda (en el mejor de los casos) con la evidencia respecto a sus resultados. Además, porque existe evidencia desde la investigación en modelos animales, que señala que ciertos tipos de estimulación pueden generar efectos no favorables en algunos dominios sociales, emocionales, o cognitivos (Brenes et al., 2016; Pietropaolo et al., 2004).

Ante este panorama, vale la pena cuestionarse: ¡cuáles son las consecuencias de exponer desde una edad temprana a un niño o niña a estos métodos de estimulación?, y ¿a cuáles aspectos del desarrollo realmente se contribuye? Producto de este trabajo se ha podido identificar que dichas preguntas han sido respondidas de forma imprecisa, o bien, desde la casuística. Adicionalmente, a estas respuestas se les asigna la cualidad de "científicas" sin que exista en realidad un cuerpo de evidencia sólido y sistemático respecto a sus efectos. 
En este contexto, iniciar por la documentación de la experiencia profesional es una invitación para aportar a la ciencia (Tapia et al., 2012) y la necesidad de compaginar la investigación básica en ciencias del desarrollo humano con sus abordajes prácticos, se torna cada vez más evidente. Finalmente, se considera que la sistematización de información sobre ET tiene que partir de conceptos más claros y específicos e incorporar la rigurosidad del método científico. Pero aún más importante, se debe partir de la evidencia existente y circunscribirse a las limitaciones de los métodos utilizados.

Esta revisión expone la falta de evidencia en los métodos de ET investigados. Las limitaciones reportadas en sus estudios, también, son comunes en el campo de la ciencia. No obstante, una de las premisas en esta última es evitar la generalización de los resultados. El sistema nervioso es sumamente versátil, complejo y susceptible a las experiencias tempranas, por lo que banalizar su entendimiento y manipulación puede concluir en resultados igualmente espurios.

\section{Limitaciones}

Los trabajos en el tema de ET suelen relacionarse con el desarrollo, implementación o análisis de intervenciones que se constituyen por conjuntos de técnicas orientadas a subsanar condiciones particulares del desarrollo o situaciones vulnerables en niños y niñas (Baker-Henningham \& López-Boo, 2014). La heterogeneidad de las propuestas planteadas como ET dificulta la búsqueda de evidencia de su efectividad, en tanto no están diseñadas para ser comparables o para aislar el efecto de una sola variable.

Dado que el ejercicio o implementación de la ET no cuenta con un ente rector al que se suscriban sus practicantes, este estudio responde a un muestreo a conveniencia que no garantiza la representatividad de todos los sectores del gremio, de modo que no es posible generalizar la información brindada en la sección de resultados para todo el territorio nacional. A esto, se suma la posibilidad de que las respuestas obtenidas puedan estar sujetas al sesgo que implica la utilización de tareas de autorreporte. Sin embargo, con el fin de disminuir esta limitación, se garantizó a las participantes el anonimato de sus respuestas al eliminar del instrumentos preguntas en las que se solicitara información que permitiera identificarlas (e.g., nombre e institución en la que trabajaba al momento de la encuesta).

Es importante destacar que no fue posible consolidar una entrevista con la Universidad Santa Paula, institución de enseñanza superior que se identificó más frecuentemente como centro de formación para la práctica de ET. Esto representa una limitación considerable, en tanto no fue posible obtener datos del número de graduadas, o bien discutir con un referente académico la información otorgada por las profesionales.

\section{Agradecimientos}

Agradecemos a las docentes y las terapeutas que brindaron información respecto a su experiencia en ET. Asimismo, se reconoce que este trabajo no hubiese sido posible sin la valiosa colaboración de las asistentes del área de Desarrollo Cognitivo del Centro de Investigación en Neurociencias de la Universidad de Costa Rica. 


\section{Referencias}

Abraham, N. M., Vincis, R., Lagier, S., Rodriguez, I., \& Carleton, A. (2014). Long term functional plasticity of sensory inputs mediated by olfactory learning. Elife, 3, e02109.

American Academy of Pediatrics (1968). The Doman-Delacato treatment of neurologically handicapped children. Developmental Medicine \& Child Neurology, 10, 243-246.

American Academy of Pediatrics. (1999).The treatment of neurologically impaired children using patterning. Pediatrics, 104(5), 1149-1151.

Atkin, L. C., Superville, T., Sawyer, R., \& Cantón, P. (1987). Paso a paso: Cómo evaluar el desarrollo y crecimiento de los niños. México: UNICEF/PAX.

Baker-Henningham, H., \& López-Boo, F. (2014). Intervenciones de estimulación infantil temprana en los países en vías de desarrollo: Lo que funciona, por qué y para quién. Económica, 60, 120-186.

Bluma, S. M., Shearer, M., Forman, A., \& Hillard, J. (1978). Guía Portage de Educación Preescolar. Barcelona: TEA.

Bonnier, C. (2008). Evaluation of early stimulation programs for enhancing brain development. Acta Paediatrica, 97(7), 853-858.

Botts, B. H., Hershfeldt, P. A., \& Christensen-Sandfort, R. J. (2008). Snoezelen ${ }^{\circledR}$ empirical review of product representation. Focus on Autism and Other Developmental Disabilities, 23(3), 138-147.

Bowlby, J. (1952). Maternal care and mental health. Geneva: WHO.

Bozic, N. (1997). Constructing the room: multi-sensory rooms in educational contexts. European Journal of Special Needs Education, 12(1), 54-70.

Brady, S., Siegel, G., Albers, R. W., \& Price, D. (Eds.). (2012). Basic neurochemistry: Principles of molecular, cellular and medical neurobiology (8th ed.). Massachusetts: Academic Press.

Bratt, B. (1989). No Time for Jello: One family's experiences with the Doman-Delacato Patterning Program. Massachusetts: Brookline Books.

Brenes, J. C., Lackinger, M., Höglinger, G. U., Schratt, G., Schwarting, R. K., \& Wöhr, M. (2016). Differential effects of social and physical environmental enrichment on brain plasticity, cognition, and ultrasonic communication in rats. Journal of Comparative Neurology, 524( 8), 1586-1607.

Bronfenbrenner, U., \& Morris, P. A. (2006). The bioecological model of human development. En R. M. Lerner (Ed.), Handbook of child development: Vol. 1. Theoretical models of human development (pp. 793-828). Hoboken, NJ: Wiley.

Chaparro, T. (2010). Método de lectura Glenn Doman. Revista Arista digital, 3, 1106-1125.

Chavarría-González, M. (1982). La estimulación temprana: apuntes sobre sus fundamentos teóricos, bases empíricas y raíces socio-históricas: elementos para una discusión. Revista de Ciencias Sociales, 23, 41-56.

Crook, S. M., Davison, A. P., \& Plesser, H. E. (2013). Learning from the past: approaches for reproducibility in computational neuroscience. In J.M. Bower (Ed.), 20 Years of Computational Neuroscience (pp. 73-102). New York, NY: Springer. 
DE LA NEUROPLASTICIDAD A LAS PROPUESTAS APLICADAS: ESTIMULACIÓN TEMPRANA Y SU IMPLEMENTACIÓN EN COSTA RICA

Cruickshank, W. (1968). Position statement on Doman-Delacato method. Exceptional Children, 34(5), 365-366.

Delacato, C. H. (1959). The treatment and prevention of reading problems: The neuro-psychological approach. Springfield, IL: Charles C. Thomas.

Deng, W., Aimone, J. B., \& Gage, F. H. (2010). New neurons and new memories: how does adult hippocampal neurogenesis affect learning and memory? Nature Reviews Neuroscience, 11(5), 339-350.

Doman, G., \& Doman, J. (2006). How to teach your baby to read: the gentle revolution. Long Island, N.Y.: Squareone Publisher.

Doman, R. J., Spitz, E. B., Zucman, E., Delacato, C. H., \& Doman, G. (1960). Children with severe brain injuries: Neurological organization in terms of mobility. JAMA, 174(3), 257-262.

Editorial Baby Star (3 de septiembre de 2009). El cerebro bebé políglota. [Archivo de video] Recuperado de: https://www. youtube.com/watch?v=Y1a8QNqUC5M.

Engert, F., \& Bonhoeffer, T. (1999). Dendritic spine changes associated with hippocampal long-term synaptic plasticity. Nature, 399(6731), 66-70.

Fernández, I., Fuente, J., \& Rueda, J. (1991). Escala Haizea-Llevant. Vitoria: Departamento de Publicaciones del Gobierno Vasco.

Fowler, S. (2008). Multisensory rooms and environments: Controlled sensory experiences for people with profound and multiple disabilities. London: Jessica Kingsley Publishers.

Frankenburg, W. K., \& Dodds, J. B. (1967). The Denver developmental screening test. The Journal of Pediatrics, 71(2), 181-191. Doi:10.1016/S0022-3476(67)80070-2

Geinisman, Y. (2000). Structural synaptic modifications associated with hippocampal LTP and behavioral learning. Cerebral Cortex, 10(10), 952-962.

Ghalambor, C. K., McKay, J. K., Carroll, S. P., \& Reznick, D. N. (2007). Adaptive versus non-adaptive phenotypic plasticity and the potential for contemporary adaptation in new environments. Functional Ecology, 21(3), 394-407.

Giese, K. P., \& Mizuno, K. (2013). The roles of protein kinases in learning and memory. Learning \& Memory, 20(10), 540-552.

Glass, G. V. (1968). Educational Piltdown Men. The Phi Delta Kappan, 50(3), 148-151.

Glass, G. V., \& Robbins, M. P. (1967). A critique of experiments on the role of neurological organization in reading performance. Reading Research Quarterly, 3(1), 5-51.

Haegele, J. A., \& Porretta, D. L. (2014). Snoezelen multisensory environment. Palaestra, 28(4), 29-32.

Haeussler, I. M., \& Marchant, T. (1985). Test de Desarrollo Psicomotor (TEPSI). Santiago: Editorial Universidad Católica.

Haggar, L. E., \& Hutchinson, R. B. (1991). Snoezelen: An approach to the provision of a leisure resource for people with profound and multiple handicaps. Journal of the British Institute of Mental Handicap (APEX), 19(2), 51-55.

Hebb, D. O. (1949). The organization of behavior. New York, NY: Wiley. 
Hernández, R.M. \& Rodríguez, S. (1987). Manual Operativo para la Evaluación y Estimulación del Crecimiento y Desarrollo del Niño. San José: EUNED.

Hogg, J., Cavet, J., Lambe, L., \& Smeddle, M. (2001). The use of 'Snoezelen' as multisensory stimulation with people with intellectual disabilities: a review of the research. Research in Developmental Disabilities, 22(5), 353-372.

Hornby, G., Atkinson, M., \& Howard, J. (2013). Controversial issues in special education. New York, N.Y.: Routledge.

Hotz, G. A., Castelblanco, A., Lara, I. M., Weiss, A. D., Duncan, R., \& Kuluz, J. W. (2006). Snoezelen: A controlled multisensory stimulation therapy for children recovering from severe brain injury. Brain Injury, 20(8), 879-888.

Hulsegge, J., \& Verheul, A. (1988). Snoezelen: Another World. Chesterfied, Derbyshire: ROMPA.

James, W. (1890). Principles of psychology. New York: Holt.

Kamerman, S. B. (2006). A global history of early childhood education and care. Background paper for EFA Global Monitoring Report 2007. Paris, France: United Nations Educational, Scientific and Cultural Organization.

Kandel, E., Schwartz, J., Jesell, T., Siegelbaim, S.A., \& Hudspeth, A.J. (Eds.). (2013). Principles of Neural Science (5th ed.). E.E.U.U.: McGraw Hill.

Kershner, J. R. (1967). An investigation of the Doman-Delacato theory of neuropsychology as it applies to trainable mentally retarded children in public schools (Tesis de Maestría inédita). Universidad de Bucknell, Estados Unidos. Recuperada de: http://files.eric.ed.gov/fulltext/ED015604.pdf

Kolb, B., Gibb, R., \& Robinson, T. E. (2003). Brain plasticity and behavior. Current Directions in Psychological Science, 12(1), 1-5.

Kolb, B., \& Whishaw, I. Q. (1998). Brain plasticity and behavior. Annual Review of Psychology, 49(1), 43-64.

Lancioni, G. E., Cuvo, A. J., \& O'Reilly, M. F. (2002). Snoezelen: an overview of research with people with developmental disabilities and dementia. Disability and Rehabilitation, 24(4), 175-184.

Leo Global Kids. (10 de julio de 2010). Soporte pedagógico. Recuperado de: http://www.leoglobalkids.com/pdfs/ soportepedagogico metodopoliglota.html

Lotan, M., \& Gold, C. (2009). Meta-analysis of the effectiveness of individual intervention in the controlled multisensory environment (Snoezelen $\mathbb{R}$ ) for individuals with intellectual disability. Journal of Intellectual \& Developmental Disability, 34(3), 207-215.

Lotan, M., \& Shapiro, M. (2005). Management of young children with Rett disorder in the controlled multi-sensory (Snoezelen) environment. Brain and Development, 27, S88-S94.

Lüscher, C., \& Malenka, R. C. (2012). NMDA receptor-dependent long-term potentiation and long-term depression (LTP/ LTD). Cold Spring Harbor Perspectives in Biology, 4, 1-16.

MacKay, D. N., Gollogly, J., \& McDonald, G. (1986). The Doman-Delacato treatment methods: I. Principles of neurological organization. The British Journal of Mental Subnormality, 32(62), 3-19.

Método bebé políglota Costa Rica. (1 febrero de 2016a). Beneficios [Sitio Web]. Recuperado de: http://bebepoliglotacostarica. com/beneficios.php 
DE LA NEUROPLASTICIDAD A LAS PROPUESTAS APLICADAS: ESTIMULACIÓN TEMPRANA Y SU IMPLEMENTACIÓN EN COSTA RICA

Método bebé políglota Costa Rica. (1 febrero de 2016b). Método Bebé Políglota [Sitio Web]. Recuperado de: http:// bebepoliglotacostarica.com/metodo.php

Mifsud, K. R., Gutiérrez-Mecinas, M., Trollope, A. F., Collins, A., Saunderson, E. A., \& Reul, J. M. (2011). Epigenetic mechanisms in stress and adaptation. Brain, behavior, and immunity, 25(7), 1305-1315.

Mora-Gallegos, A., Rojas-Carvajal, M., Salas, S., Saborío-Arce, A., Fornaguera-Trías, J., \& Brenes, J. C. (2015). Agedependent effects of environmental enrichment on spatial memory and neurochemistry. Neurobiology of Learning and Memory, 118, 96-104.

Moser, M. B., Trommald, M., \& Andersen, P. (1994). An increase in dendritic spine density on hippocampal CA1 pyramidal cells following spatial learning in adult rats suggests the formation of new synapses. Proceedings of the National Academy of Sciences, 91(26), 12673-12675.

Nasser, K., Cahana, C., Kandel, I., Kessel, S., \& Merrick, J. (2004). Snoezelen: Children with intellectual disability and working with the whole family. The Scientific World Journal, 4, 500-506.

Ortiz-Alonso, T, Hall, C. V., \& García, J. M. S. (2013). Localización de fuentes cerebrales en niños generadas por estimulación multilingüística simultánea. Participación educativa, 2(3), 175-186.

Palacios, J., Marchesi, A. \& Coll, C. (2006). Desarrollo psicológico y educación: Psicología evolutiva. Madrid: Alianza.

Pietropaolo, S., Branchi, I., Cirulli, F., Chiarotti, F., Aloe, L., \& Alleva, E. (2004). Long-term effects of the periadolescent environment on exploratory activity and aggressive behaviour in mice: social versus physical enrichment. Physiology \& Behavior, 81(3), 443-453.

Piper, M. C., \& Darrah, J. (1994). Alberta Infant Motor Scale (AIMS). Philadelphia, PA: Saunders.

Rojas, L. (10 de setiembre de 2014). Hospital de Niños tiene una novedosa sala que permite fortalecer los sentidos de los menores. CRHoy.com. Recuperado de http://www.crhoy.com/ hospital-de-ninos-tiene-una-novedosa-sala-que-permite-fortalecer-los-sentidos-de-los-menores/

Rosenzweig, M. R., Bennett, E. L., \& Diamond, M. C. (1972). Brain changes in response to experience. Scientific American, $226(2), 22-29$.

Rutter, M. (2007). Gene-environment interdependence. Developmental Science, 10(1), 12-18.

Sale, A., Berardi, N., \& Maffei, L. (2014). Environment and brain plasticity: Towards an endogenous pharmacotherapy. Physiological Reviews, 94(1), 189-234.

Shapiro, M., Melmed, R. N., Sgan-Cohen, H. D., Eli, I., \& Parush, S. (2007). Behavioural and physiological effect of dental environment sensory adaptation on children's dental anxiety. European Journal of Oral Sciences, 115(6), 479-483.

Shapiro, M., Parush, S., Green, M., \& Roth, D. (1997). The efficacy of the "Snoezelen" in the management of children with mental retardation who exhibit maladaptive behaviours. The British Journal of Development Disabilities, 43(85), 140-155.

Shonkoff, J.P., \& Meisels, S.J. (2000). Prefacio. En: J. P. Shonkoff, \& S. J. Meisels (Eds.), Handbook of Early Childhood Intervention, (p.p. xvii-xviii). Cambridge University Press. 
Stephenson, J., \& Carter, M. (2011). Use of multisensory environments in schools for students with severe disabilities: Perceptions from schools. Education and Training in Autism and Developmental Disabilities, 46(2), 276-290.

Stone, M., \& Pielstick, N. L. (1969). Effectiveness of Delacato treatment with kindergarten children. Psychology in the Schools, 6(1), 63-68.

Sweatt, J. D. (2009). Experience-dependent epigenetic modifications in the central nervous system. Biological Psychiatry, 65(3), 191-197.

Tapia, J., Carmiol, A. M., \& Rosabal-Coto, M. (2012). La psicología del desarrollo en Costa Rica: alcances y perspectivas futuras. Revista Costarricense de Psicología, 31(1-2), 101-121.

Terré, O. (2010). Cómo potenciar la inteligencia del niño: Método Point [Sitio Web]. Recuperado de: http://www. orlandoterre.com/met1.html

The Institutes for the Achievement of Human Potential. (8 de noviembre de 2016). Mayor Victories: Achieved by 3024 Brain-Injured Children [Sitio Web]. Recuperado de: https://www.iahp.org/results/.

Vergara-Díaz, G., Martínez-Galán, M., Martínez-Sahuquillo, M.E., \& Echevarría-Ruiz, C. (2011). Eficacia del método de los institutos para el Logro del Potencial Humano (Doman-Delacato) en pacientes con parálisis cerebral infantil. Rehabilitación, 45(3), 256-260.

Wachelke, J. F. R., Natividade, J. C., Faggiani, R. B., \& De Andrade, A. L. (2004). Contribuições e limitações do Método Doman-Delacato no contexto da edução especial. Revista Brasileira de Educação Especial, 10(3), 309-320.

Weisberg, D. S., Keil, F. C., Goodstein, J., Rawson, E., \& Gray, J. R. (2008). The seductive allure of neuroscience explanations. Journal of Cognitive Neuroscience, 20(3), 470-477.

Wepman, J. M. (1958). Auditory discrimination test. Chicago, IL.: Language Research Associates.

Ziring, P. R., Cooley, W. C., Kastner, T. A., Kummer, M. E., González, D. P. L., Quint, R. D., ... \& Sandler, A. D. (1999). American Academy of Pediatrics. Committee on Children with Disabilities. The treatment of neurologically impaired children using patterning. Pediatrics, 104(5 Pt 1), 1149-1151. 


\section{Sobre las autoras y los autores:}

Johanna Sibaja-Molina. Es licenciada en Psicología y egresada de la Maestría en Ciencias Cognoscitivas de la Universidad de Costa Rica (UCR). Labora como profesora en la Escuela de Psicología y de Formación Docente de la UCR. Funge como investigadora en el área de Desarrollo Cognitivo del Centro de Investigación en Neurociencias (CIN) de la UCR. Coordina el Programa de Educación Continua del CIN, cuyo objetivo es la divulgación científica. Sus proyectos de investigación se vinculan con neurodesarrollo cognitivo, en particular el tema de dificultades de aprendizaje en escolares. Ha participado en congresos como expositora de temas como las funciones ejecutivas y la relación entre neurociencias y educación. Cuenta con varias publicaciones académicas.

Tracy Sánchez-Pacheco. Obtuvo el bachillerato en Psicología en la Universidad de Costa Rica. Actualmente, es candidata al Posgrado en Ciencias Cognoscitivas de la Universidad de Costa Rica. Se desempeña como investigadora en el grupo de cognición social del Instituto de Investigaciones Psicológicas y en el grupo de desarrollo cognitivo del Centro de Investigación en Neurociencias de la Universidad de Costa Rica. Sus áreas de interés son las cogniciones sociales, las funciones ejecutivas y la electroencefalografia.

Mijail Rojas-Carvajal. Es licenciado en Psicología. Trabaja como investigador del Centro de Investigación en Neurociencias de la Universidad de Costa Rica. Su labor se orienta al estudio de los efectos de los ambientes tempranos sobre la habituación, la memoria espacial y el autoacicalamiento en roedores. Además, colabora en distintas investigaciones sobre el efecto de las drogas psicoestimulantes y el estrés sobre el sistema nervioso central. Actualmente, es candidato a la Maestría en Ciencias Cognoscitivas de la Universidad de Costa Rica, y orienta su trabajo de graduación al estudio de los mecanismos conductuales y genéticos subyacentes de la memoria asociativa y no asociativa.

Jaime Fornaguera-Trías. Se tituló como bachiller en Biología y máster en Fisiología de la Universidad de Costa Rica y doctor en Neurofisiología de la Universidad Heinrich Heine, Düsseldorf, Alemania. Ha laborado como Director del Centro de Investigación en Neurociencias, Universidad de Costa Rica, Profesor de grado y posgrado de la Universidad de Costa Rica. Se ha destacado como miembro fundador de la Federación Latinoamericana y del Caribe de Neurociencias y representante para Centroamérica de dicha Federación, Revisor de publicaciones nacionales e internacionales en el área de las Neurociencias y de la Educación. Cuenta con más de 50 publicaciones en revistas indexadas. Conferencista en temas de Neurociencias y de Educación

Publicado en línea: 16 de diciembre de 2016 